\title{
Investigating the effect of some parameters of the channel on the characteristics of tunneling carbonnanotube field-effect transistor
}

\author{
Najmeh Valed Karimi ${ }^{1}$ [ $\cdot$ Yaghoub Pourasad $^{2}$
}

Received: 30 June 2015/ Accepted: 25 June 2016/Published online: 25 August 2016

(c) The Author(s) 2016. This article is published with open access at Springerlink.com

\begin{abstract}
This paper studies p-i-n tunneling carbon nanotube field-effect transistor to investigate the effect of various parameters of the channel on the characteristics of tunneling carbon nanotube field-effect transistor. Tunneling carbon nanotube field-effect transistor (T-CNTFET) has been simulated using non-equilibrium Green's function (NEGF), and the transmission was conducted through inelastic scattering. Besides the evaluation of device performance, various parameters of the channel were also compared. One of the parameters is considered as the variable, while other parameters of the channel are constant. Then, improved characteristics were discussed by selection of some channel parameters. T-CNTFET with CNT $(10,0)$ with oxide thickness $=1 \mathrm{~nm}$ shows reduced sub-threshold swing (18 mV/decade).
\end{abstract}

Keywords Channel diameter · Channel length (L) - Gate oxide thickness · Non-equilibrium Green's function (NEGF) - Tunneling carbon nanotube field-effect transistor (T-CNTFET)

Najmeh Valed Karimi

n.karimi6585@yahoo.com

Yaghoub Pourasad

y.pourasad@uut.ac.ir

1 Electrical Engineering Department, Islamic Azad University, Rasht Branch, Rasht, Guilan, Iran

2 Electrical Engineering Department, Urmia University of Technology, Urumia, Iran

\section{Introduction}

While technology-based silicon will reveal its limitations, some emerging devices are investigated to find appropriate substitution to silicon. Carbon nanotube FETs (CNTFETs) are illustrated to replace silicon in the future [1]. Carbon nanotubes were explored by Sumio Iijima in 1991 [2]. Carbon nanotubes have properties, such as caring high current, ballistic transport, mechanical stability, and dynamic load, which make them appropriate substances to replace silicon as the channel of field-effect transistors. Ballistic transport in p-type CNTFETs using quasi-planar gate geometries and higher on-currents to MOSFET has been proved by experiments $[3,4]$. Cylindrical carbon nanotube with gate-all-around (GAA) leads to an increase in miniaturization and also provides electrostatic control of the gate [5]. There is charge pile-up in the channel CNTFET, which leads to the increase in the off-current and lowers the $I_{\mathrm{ON}} / I_{\mathrm{OFF}}$ ratio. To overcome this problem, a gate-controlled tunneling device (T-CNTFET) is offered. A steep inverse sub-threshold slope and the better controlled off-current were obtained by T-CNTFETs. In T-CNTFET, the increase in the potential difference between the gate and the drain contact leads to strong band-bending near the drain contact [6]. The T-CNTFET has been simulated by self-consistent solution between the Poisson and Schrodinger equations [3]. As can be found from [3], to suppress the ambipolar behavior of CNTFET and to improve the performance of this device, the asymmetric double-halo doping has been applied in CNTFET. In addition, for this purpose, CNTFETs with graded double-halo channel are presented as in [7]. Transmission is considered ballistic in both [3] and [7]. In this paper, we investigate the effect of changing a channel parameter on characteristics of $\mathrm{T}$-CNTFET, provided that the other channel parameters 
remain constant. The investigated channel parameters include channel length $(L)$, channel diameter, and gate oxide thickness. Finally, on-current (Ion), tunneling current, on/off-current ratio $\left(I_{\mathrm{ON}} / I_{\mathrm{OFF}}\right)$, and sub-threshold swing (SS) were the characteristics investigated in this research.

\section{Device structure}

Figure 1 demonstrates the illustration of T-CNTFET structure. The CNT is made of a zigzag $(16,0)$ of $0.63-\mathrm{nm}$ radius and length of $L=15 \mathrm{~nm}$. A coaxial gate is placed around the channel region of the nanotube. The dielectric layer $\mathrm{HfO}_{2}$ has $2 \mathrm{~nm}$ thickness, and the relative dielectric constant is $\varepsilon_{\mathrm{r}}=16$. $\mathrm{HfO}_{2}$ separates the gate from CNT $2 \mathrm{~nm}$. T-CNTFET included in p-doped source and n-doped drain regions with $30 \mathrm{~nm}$ length which have been doped by $2 \mathrm{~nm}^{-1}$ doping.

\section{Computational method}

The proposed device is simulated by the self-consistent solution of the poison and Schrodinger equations within the NEGF formalism. The nanoscale system is simulated by the NEGF method out of equilibrium. The electrostatic potential is obtained by the Poisson equation to compute the Hamiltonian of the system [3]. Carbon nanotube is coaxially gated, so symmetric characteristics of cylindrical coordinates create the potential, and the charge is constant around the nanotube. Consequently, the Poisson equation is essentially a $2 \mathrm{D}$ problem along the tube ( $z$-direction) and the redial direction ( $r$-direction). Eigen states around the tube circumferential direction (modes) are plane wave vectors satisfying the periodic boundary condition [7]:

$\nabla^{2} \boldsymbol{U}\left(\boldsymbol{r}, \boldsymbol{Z}_{\boldsymbol{j}}\right)=-\rho\left(\boldsymbol{r}, \boldsymbol{Z}_{\boldsymbol{j}}\right) / \varepsilon_{0} \varepsilon_{\boldsymbol{r}}$,

where $\boldsymbol{U}_{\boldsymbol{J}}(\boldsymbol{r}, \boldsymbol{z}), \varepsilon$, and $\mathrm{p}\left(\boldsymbol{r}, \boldsymbol{Z}_{j}\right)$ are the electrostatic potential, the constant dielectric, and the net charge density distribution, which include dopant density as well, respectively. The retarded Green's function for the device in matrix form is computed as

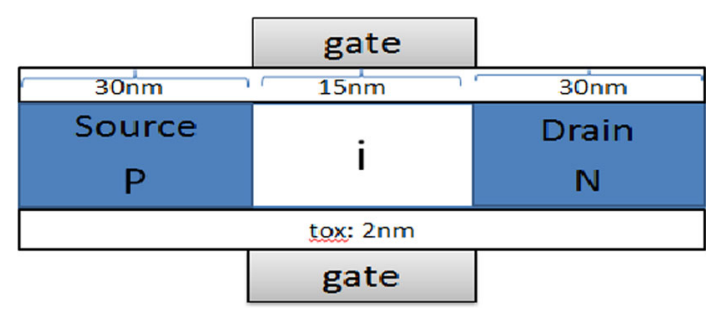

Fig. 1 Schematic cross-sectional view of the coaxial T-CNTFET
$G(E)=\left[\left(E+i \eta^{+}\right) I-H-\sum_{\mathrm{S}}-\sum_{\mathrm{D}}\right]^{-1}$,

where $\sum_{\mathrm{S}}$ and $\sum_{\mathrm{D}}$ are the self-energies of the source and the drain, respectively. $\eta^{+}, E, I$, and $H$ are an infinitesimal positive value, the energy, the identity matrix, and the Hamiltonian of the CNT, respectively:

$H_{q}=\left[\begin{array}{ccccccc}U_{1} & b_{2 q} & 0 & 0 & & & \\ b_{2 q} & U_{2} & t & 0 & & 0 & \\ 0 & t & U_{3} & b_{2 q} & & & \\ & & & & \ddots & t & 0 \\ & 0 & & t & U_{n-1} & b_{2 q} \\ & & & & 0 & b_{2 q} & U_{n}\end{array}\right]$,

where $b_{2 q}=2 t \cos (\pi q / n), t \approx 3 \mathrm{eV}$, and $N$ are the nearest neighbor hopping parameter and the total number of carbon rings along the device, respectively. The diagonal elements $U_{J}$ are assumed as the no-site electrostatic potential along the tube surface as in [8]. The self-energy matrix can be interpreted as a boundary condition of the Schrodinger equation. In this paper, we investigated a self-energy for semi-infinite that leads to boundary conditions, which enables to consider the CNT as connected to infinitely long CNTs at its ends. The source self-energy function $\sum_{\mathrm{s}}$ has all its entries zero except for the $(1,1)$ factor:

$$
\begin{aligned}
\sum_{\mathbf{s}}(\mathbf{1}, \mathbf{1})= & \frac{\left(\mathbf{E}-\mathbf{U}_{1}\right)^{2}+\mathbf{t}^{2}+\mathbf{b}_{2 \mathbf{q}}^{2}}{2\left(\mathbf{E}-\mathbf{U}_{1}\right)} \\
& \pm \frac{\sqrt{\left[\left(\mathbf{E}-\mathbf{U}_{1}\right)^{2}+\mathbf{t}^{2}+\mathbf{b}_{2 \mathbf{q}}\right]^{2}-4\left(\mathbf{E}-\mathbf{U}_{1}\right)^{2} \mathbf{t}^{2}}}{2\left(\mathbf{E}-\mathbf{U}_{1}\right)} .
\end{aligned}
$$

In addition, $\sum_{\mathrm{D}}$ has only its $(N, N)$ element nonzero, and it is given by an equation similar to $\mathbf{E}_{q}$ with $\mathbf{U}_{1}$ displaced by $\mathbf{U}_{N}$ as in [8]. The two-dimensional nanotube lattice of a $(n, 0)$ zigzag CNT was transformed to $n$ decoupled one dimensional modes by doing a basis transform from the real space to the mode space in the circumferential direction. Under typical bias conditions, the few modes that are relevant to electronic transport are treated. For this mode, the charge density is calculated by integrating the local density-of-states (LDOS) over energy:

$$
\begin{aligned}
Q(Z) & =(-q) \int_{+\infty}^{-\infty} \mathrm{d} E \cdot \operatorname{sgn}\left[E-E_{N}(z)\right] \\
& \left\{D_{\mathrm{is}}(E, z) f\left(\operatorname{sgn}\left[E-E_{N}(z)\right]\left(E-E_{\mathrm{FS}}\right)\right)\right. \\
& \left.+D_{\mathrm{iD}}(E, z) f\left(\operatorname{sgn}\left[E-E_{N}(z)\right]\left(E-E_{\mathrm{FS}}\right)\right)\right\}
\end{aligned}
$$

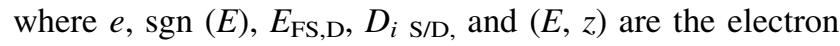
charge, the sign function, the source (drain) Fermi level, the LDOS due to the source (drain) contact as computed by 
the NEGF method, respectively. In this paper, we have used single-pi model for all simulations; nanotube conduction and valence bands are considered symmetric, so the charge neutrality level, $E_{N}(z)$, lies at the middle of bandgap. The potentials at the source/drain and gate electrodes are assumed fixed as the boundary conditions. The iteration between the atomistic quantum transport equation and the electrostatic equation continues until self-consistency is obtained, and then, Landaur-Buttiker formula computes the source-drain current:

$I=2 q / h \int T(E)\left[F\left(E-E_{\mathrm{FS}}\right)-\left(E-E_{\mathrm{FD}}\right)\right] \mathrm{d} E$.

In this formula, $T(E)$ is transmission coefficient [7].

\section{Results and discussion}

Figure 2 shows the behavior of T-CNTFET clearly. The carriers can tunnel from the source to the drain provided that conduction band $\left(E_{\mathrm{C}}\right)$ in the channel is higher than the valence band $\left(E_{\mathrm{V}}\right)$ of the source. For the equilibrium condition, the whole length of the channel region acts as a tunneling barrier. On the other hand, when $E_{\mathrm{C}}$ in the channel is lower than the $E_{\mathrm{V}}$ in the source, the height of the tunneling barrier will decrease at the source-channel side and as a result of band-to-band tunneling current (applied $V_{\mathrm{DS}}>0$ ). The phonon-induced states can create further tunneling paths of carriers that lead to higher tunneling current under scattering as in [6]. Thus, the transmission was assumed with inelastic scattering. Figure 3 shows that when the device is on, the drain current saturates at high $V_{\mathrm{DS}}$. But increasing $V_{\mathrm{DS}}$ would finally result in tunneling at

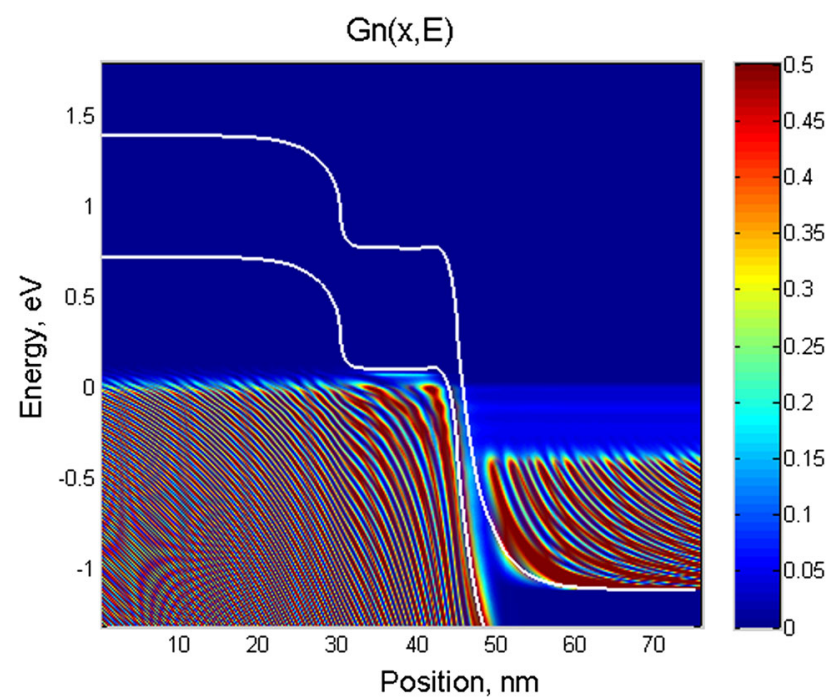

Fig. 2 Color-scaled plot for the number of electrons per-unit energy along the CNT axis for T-CNTFET; the biasing conditions are $V_{\mathrm{GS}}=-0.6 \mathrm{~V}$ and $V_{\mathrm{DS}}=0.4 \mathrm{~V}$

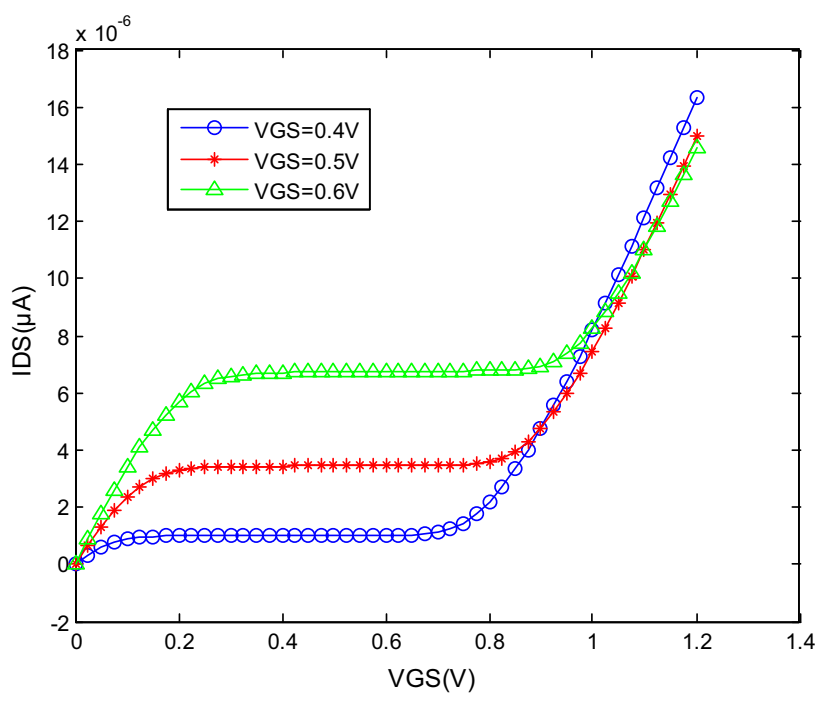

Fig. 3 Output characteristics of the T-CNTFET structure at different gate voltages

the drain-channel side. This ambipolar behavior limits the performance of the device. As $V_{\mathrm{GS}}$ increases, the bending of the bands at the source side will increase more and consequently causes tunneling barrier thinner and rises the transmission $\mathrm{r}$ as well. It is effective less than $60 \mathrm{mV} /$ decade swing, and leads to increase on-current essentially (Fig. 4). On the other hand, applying a negative $V_{\mathrm{GS}}$ leads to band-to-band tunneling at the drain-channel side, and results in ambipolar conduction. There is the current minimum at $V_{\mathrm{GS}}=V_{\mathrm{DS}} / 2$ for symmetric source/drain doping. Figure 4 shows $I_{\mathrm{DS}}-V_{\mathrm{GS}}$ characteristics for T-CNTFET at different $V_{\mathrm{DS}}$ values. As can be observed from Fig. 4, the drain current saturates at high $V_{\mathrm{DS}}$ for T-CNTFET.

The ambipolar behavior leads to the high off-current, also the sub-threshold slope and the on-current increase. Due to the rise of off-current, the $I_{\mathrm{ON}} / I_{\mathrm{OFF}}$ ratio reduces

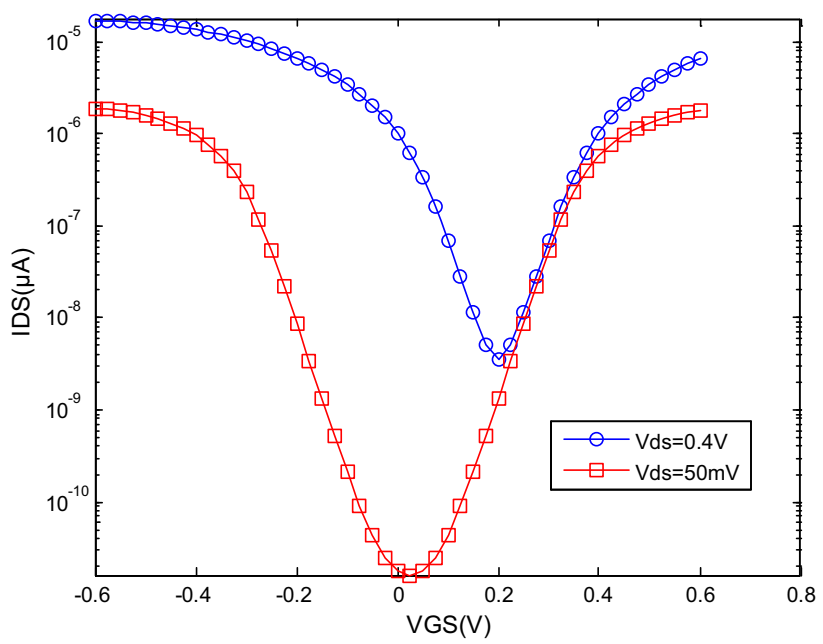

Fig. 4 Transconductance characteristics of the T-CNTFET structure at $V_{\mathrm{DS}}=0.4 \mathrm{~V}$ and $50 \mathrm{mV}$ 
subsequently. Therefore, we investigate various channel parameters, to achieve improved characteristics. Then, with choosing some channel parameters, we improve some characteristics of T-CNTFET.

The diameter of CNT is inversely proportional to bandgap energy. The bandgap energy is the minimum energy needed to break a covalent bond in the semiconductor crystal, so as to free an electron for conduction. This means that lower bandgap energy creates a better conduction material $[9,10]$.

Diameter CNT is acquired from the following equation:

$D_{\mathrm{CNT}}=\frac{\sqrt{3}}{\pi} a_{0} \sqrt{n^{2}+m^{2}+m n}$.

That $a_{0}=0.425 \mathrm{~nm}$ is carbon-to-carbon distance, $n$ and $m$ are described CNT diameter and zero, respectively. The threshold voltage $\left(V_{\mathrm{th}}\right)$ is described as the voltage required to switch on a transistor. A threshold voltage of the intrinsic CNT channel can be approximated as the halfbandgap that is an inverse function of the diameter:

$V_{\mathrm{th}}=\frac{\mathbf{E g}}{2 \mathbf{e}}=\frac{\sqrt{3}}{3} \frac{\mathbf{a} V_{\pi}}{3 \mathbf{e} D_{\mathrm{CNT}}}$,

where $\mathbf{a}=2.49 \AA$ is the carbon-to-carbon distance, $V_{\pi}=3.033 \mathrm{eV}$ is the carbon $\pi-\pi$ bond energy in the tight bonding model, $\mathbf{e}$ is the unit electron charge, and $\boldsymbol{D}_{\mathbf{C N T}}$ is the CNT diameter [10]. Table 1 shows that as CNT diameter increases, threshold voltage decreases and leads to more conduction. The threshold voltage is defined for various values of $n$, as shown in Table 1. Electronics industry is looking for more reductions in the size of MOSFETs. The MOSFET channel length is a measure for the size of its other parameters. The final proposal is to achieve the channel length $10 \mathrm{~nm}$ and the less. In this structure, coaxial gate covers all around channel thus are defined as one of the channel parameters. Figure 5 is investigated with various CNT diameter at a constant $L=15 \mathrm{~nm}$ and oxide thickness $=2 \mathrm{~nm}$, different channel length at the constant channel diameter $(n=16)$ and oxide thickness $=2 \mathrm{~nm}$, and also different oxide thickness at the fixed $L=15 \mathrm{~nm}$ and CNT $(16,0)$ for $I_{\mathrm{DS}}-V_{\mathrm{GS}}$ of the T-CNTFET. As can be seen from Fig. 5, on-current and tunneling current increase due to increase in CNT diameter at the constant $L=15 \mathrm{~nm}$ and oxide thickness $=2 \mathrm{~nm}$. The on-current and tunneling current increase due to the

Table 1 Threshold voltages for various channel diameters of CNTFET

\begin{tabular}{lll}
\hline$\sim V_{\mathrm{th}}(\mathrm{V})$ & $\sim D_{\mathrm{CNT}}$ & $n$ \\
\hline 0.55 & 0.7837 & 10 \\
0.4 & 1.0118 & 13 \\
0.35 & 1.2539 & 16 \\
0.3 & 1.489 & 19 \\
\hline
\end{tabular}

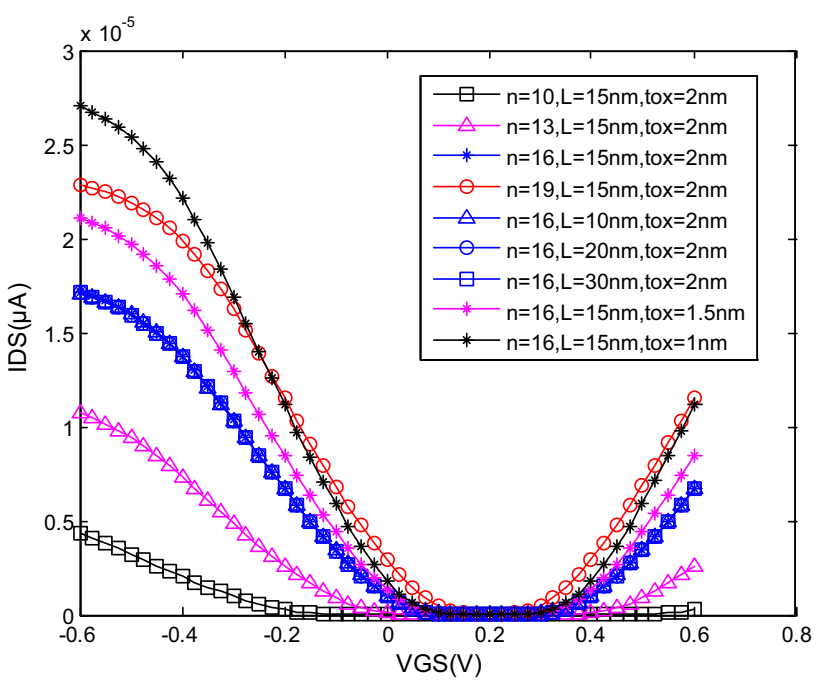

Fig. 5 Transconductance characteristics of T-CNTFET with various channel parameters at $V_{\mathrm{DS}}=0.4 \mathrm{~V}$

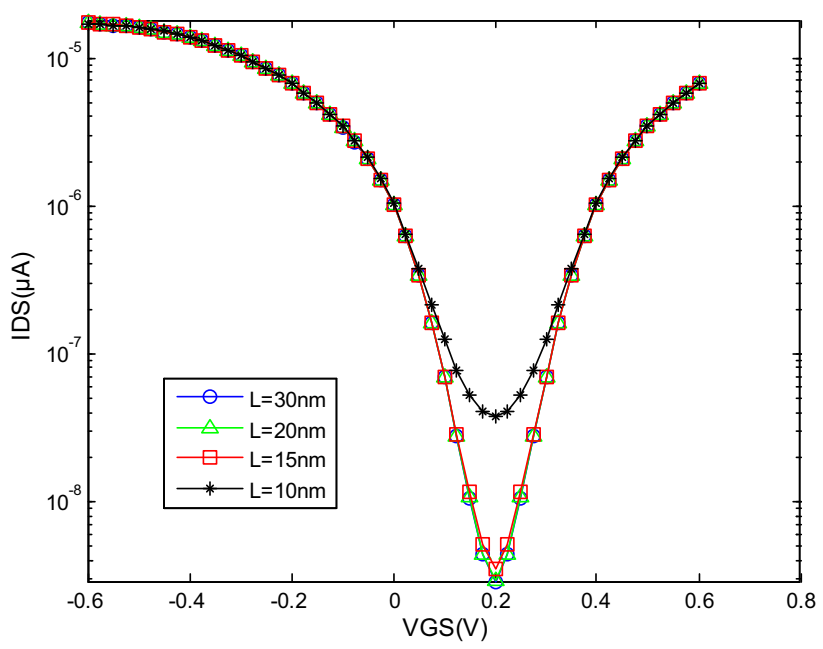

Fig. 6 Transconductance characteristics of T-CNTFET with various channel lengths at $V_{\mathrm{DS}}=0.4 \mathrm{~V}$

fact that the CNT diameter is increased, so the bandgap energy lowers which result in the higher conduction. In addition, Fig. 5 demonstrates, for constant CNT diameter and CNT length, reduction oxide thickness leads to the increase in $I_{\mathrm{ON}}$ and tunneling current. Thinner gate oxide results in the reduction of gate-source fringing. Due to this reduction, the height of the barrier energy decreases at both source-channel and drain-channel sides which result in lower tunneling barriers and $I_{\mathrm{ON}}$. Gate-drain fringing can repress the ambipolar conduction but degenerates highfrequency operation by increased fringe capacitances. As CNT diameter and gate oxide thickness are constant, $I_{\mathrm{ON}}$ and tunneling current do not vary by reducing channel length (for inelastic scattering transport). For further observation, we investigated $I_{\mathrm{DS}}-V_{\mathrm{GS}}$ figure for the range 


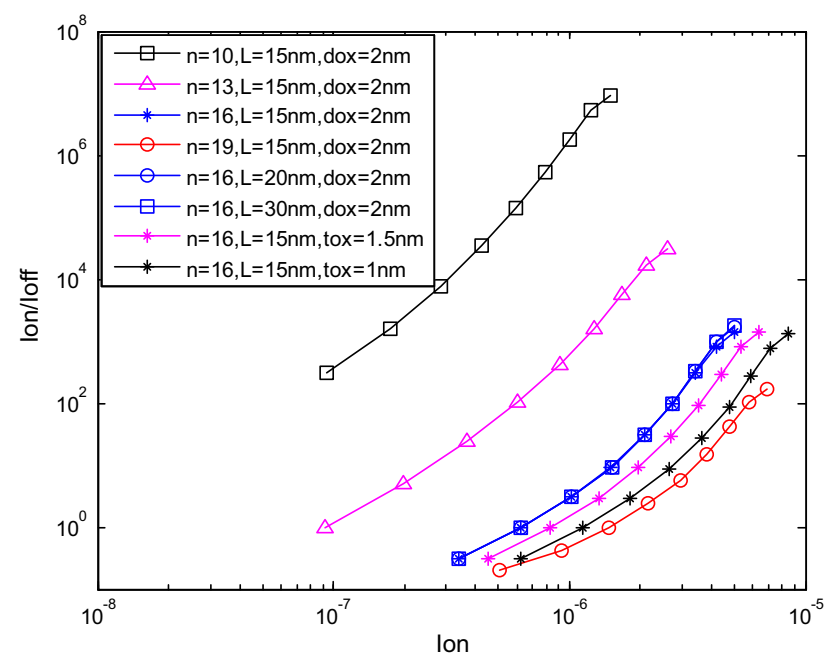

Fig. $7 I_{\mathrm{ON}} / I_{\mathrm{OFF}}$ ratio as a function of $I_{\mathrm{ON}}$ for T-CNTFET with different channel parameters

of the channel lengths in Fig. 6, separately. Figure 6 demonstrates that for $L=15,20$, and $30 \mathrm{~nm}$, the reduction of channel length does not reduce $I_{\mathrm{ON}}$ but $I_{\mathrm{OFF}}$ increases, because direct tunneling from source to drain for shortchannel length $\left(L_{\mathrm{G}} \leq 10 \mathrm{~nm}\right)$. As can be found from Fig. 5, the largest $I_{\mathrm{ON}}$ and tunneling current are observed by T-CNTFET with CNT $(16,0), L=15 \mathrm{~nm}$, and oxide thickness $=1 \mathrm{~nm}$. In addition, smallest $I_{\mathrm{ON}}$ and tunneling current are seen by device with CNT $(10,0), L=10 \mathrm{~nm}$, and oxide thickness $=2 \mathrm{~nm}$.

For further comparison, we evaluated the $I_{\mathrm{ON}} / I_{\mathrm{OFF}}$ ratio as a function of $I_{\mathrm{ON}}$ at a $V_{\mathrm{DS}}=0.4 \mathrm{~V}$. Figure 7 shows the $I_{\mathrm{ON}} / I_{\mathrm{OFF}}$ characteristics for T-CNTFET with all various parameters of the channel. As can be observed from Fig. 7 with decreasing CNT diameter, $I_{\mathrm{ON}} / I_{\mathrm{OFF}}$ ratio increases. The reason of this increase is that with reduction of the CNT diameter, the off-current reduced, it leads to higher $I_{\mathrm{ON}} / I_{\mathrm{OFF}}$ ratio, which causes an increased $I_{\mathrm{ON}} / I_{\mathrm{OFF}}$ ratio for the T-CNTFET with smaller channel diameter. For CNTs $(10,0),(13,0),(16,0)$, and $(19,0), I_{\mathrm{ON}} / I_{\mathrm{OFF}}$ ratio is $>10^{6}$, $>10^{4},>10^{3}$, and $>10^{2}$, respectively. Furthermore, Fig. 7 illustrates for the range of oxide thickness include in $1,1.5$, and $2 \mathrm{~nm}$ provided that other parameters are constant. ON/ OFF-current ratio is constant and nearly $>10^{3}$. In addition, it demonstrates for $L=15,20$, and $30 \mathrm{~nm}, I_{\mathrm{ON}} / I_{\mathrm{OFF}}$ ratio is nearly constant, and $>10^{3}$ which is due to fixed $I_{\mathrm{ON}}$ and $I_{\mathrm{OFF}}$ for various channel lengths. For further evaluation, Fig. 8 was investigated. As can be seen from Fig. 8 due to large off-current $L=10 \mathrm{~nm}$ compared to other channel length, $I_{\mathrm{ON}} / I_{\mathrm{OFF}}$ is decreased and its value is $>10^{2}$. The largest $I_{\mathrm{ON}} / I_{\mathrm{OFF}}$ ratio are observed by T-CNTFET with CNT $(10,0), L=15 \mathrm{~nm}$, and oxide thickness $=2 \mathrm{~nm}$. The sub-threshold swing (SS) is defined as the change in

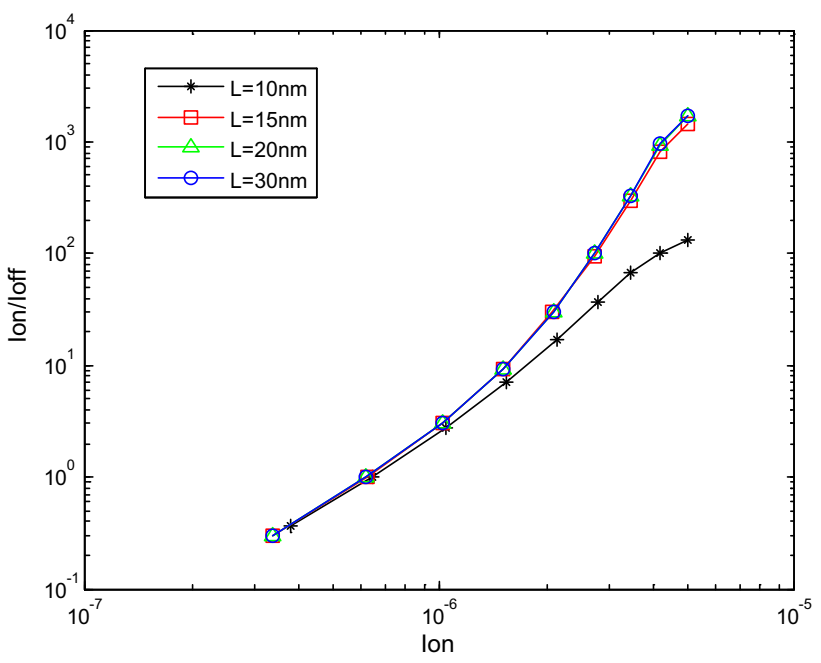

Fig. $8 I_{\mathrm{ON}} / I_{\mathrm{OFF}}$ ratio as a function of $I_{\mathrm{ON}}$ for T-CNTFET with different channel lengths, $n=16$, and oxide thickness $=2 \mathrm{~nm}$

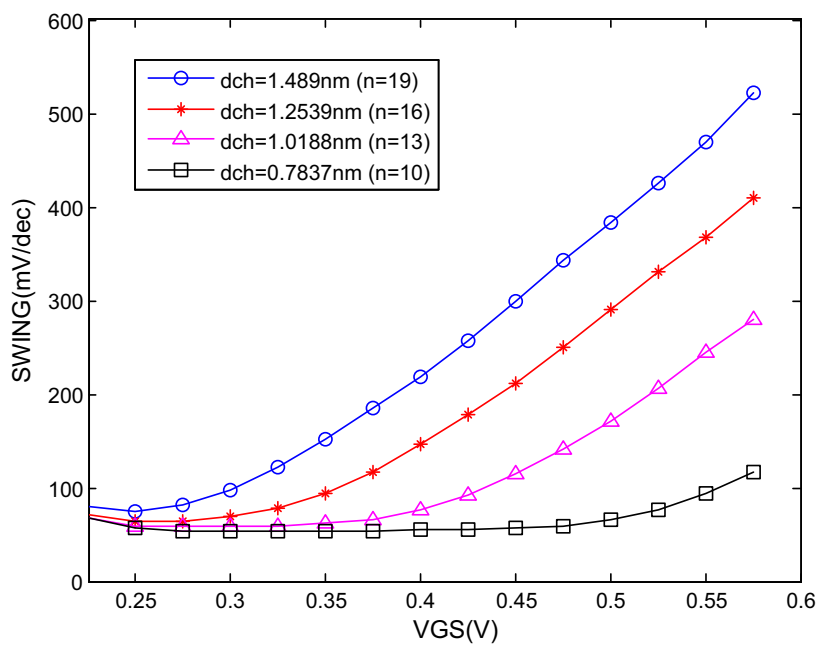

Fig. 9 Sub-threshold swing as a function of the gate-source voltage with various channel diameters, $L=15 \mathrm{~nm}$, and oxide thickness $=2 \mathrm{~nm}$

gate bias needed to change the sub-threshold drain current by one decade, as given by:

$\mathbf{S S} \mid \boldsymbol{V}_{\mathbf{G S}}=\left(\partial\left(\frac{\log \boldsymbol{I}_{\mathbf{D S}}}{\partial \mathbf{V g S} \mid \boldsymbol{V}_{\mathbf{G S}}}\right)\right)^{-1}$.

$\boldsymbol{I}_{\mathbf{D S}}$ and $\boldsymbol{V}_{\mathbf{G S}}$ are descried as drain-source current and gate-source voltage, respectively $[5,11]$. Figure 9 demonstrates sub-threshold swing as a function of the gatesource voltage for variation in CNT diameter, provided that other channel parameter is constant. As can be found from Fig. 9 as channel diameter decreases, the band gap energy will increase more, making the tunneling barrier increasingly ticker and leads to the decrease in the drain 


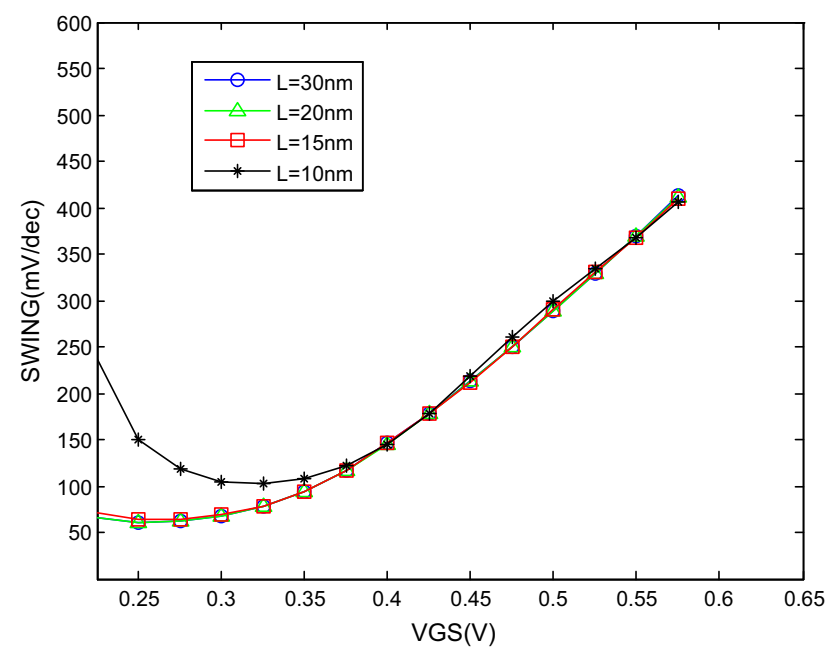

Fig. 10 Sub-threshold swing as a function of the gate-source voltage with various channel lengths, $n=16$, and oxide thickness $=2 \mathrm{~nm}$

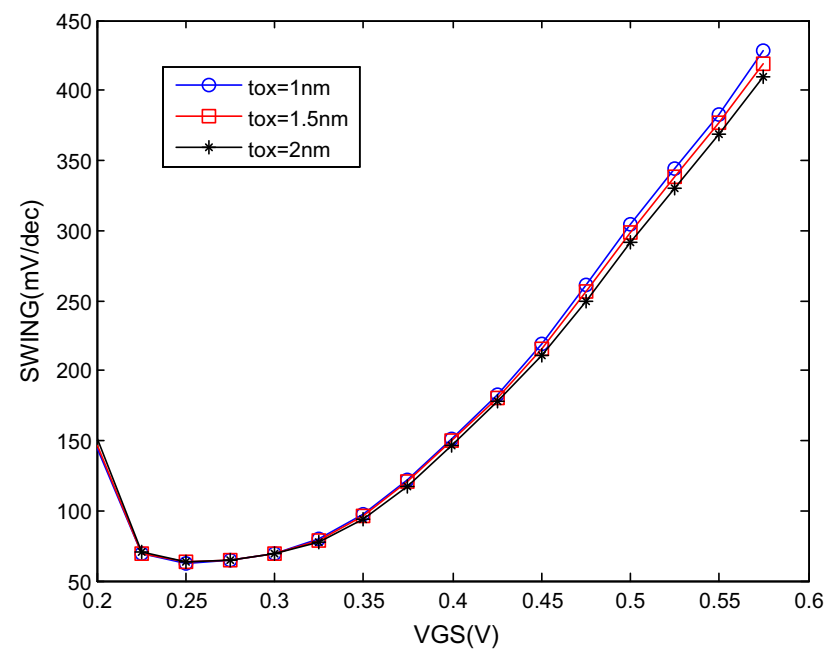

Fig. 11 Sub-threshold swing as a function of the gate-source voltage with various channel diameters, $L=15 \mathrm{~nm}$, and oxide thickness $=2 \mathrm{~nm}$

transconductance also threshold voltage increases and results in on-current/sub-threshold swing decrease/ increase.

This causes less than $60 \mathrm{mV} /$ decade sub-threshold swing. For CNTs $(10,0)$ and $(13,0)$, sub-threshold swing is 53 and $56 \mathrm{mV} /$ decade, respectively. In addition, for CNTs $(16,0)$ and $(19,0)$, sub-threshold swing is not achieved less than $60 \mathrm{mV} /$ decade swing. Reduction of oxide thickness does not affect sub-threshold swing. Figure 10 shows that subthreshold swing is constant while channel length is varied. Swing sub-threshold is $60 \mathrm{mV} / \mathrm{dec}$ ade for the range of the channel lengths 30,20 , and $15 \mathrm{~nm}$, respectively. For $\mathrm{L}=10$ $\mathrm{nm}$, sub-threshold swing is not observed less than $60 \mathrm{mV} /$ decade which that is undesirable. Reason of this increase is high off-current for the channel length $10 \mathrm{~nm}$. Figure 11

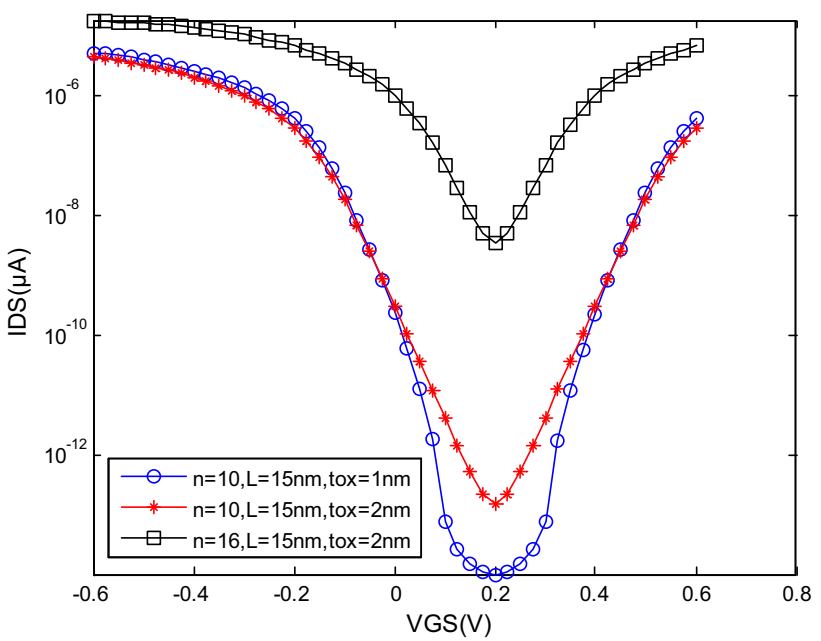

Fig. 12 Comparision transconductance characteristics of T-CNTFET for $\operatorname{CNT}(10,0)$, tox $=1 \mathrm{~nm}$ and $(16,0)$, and tox $=2 \mathrm{~nm}$ at $V_{\mathrm{DS}}=0.4 \mathrm{~V}$

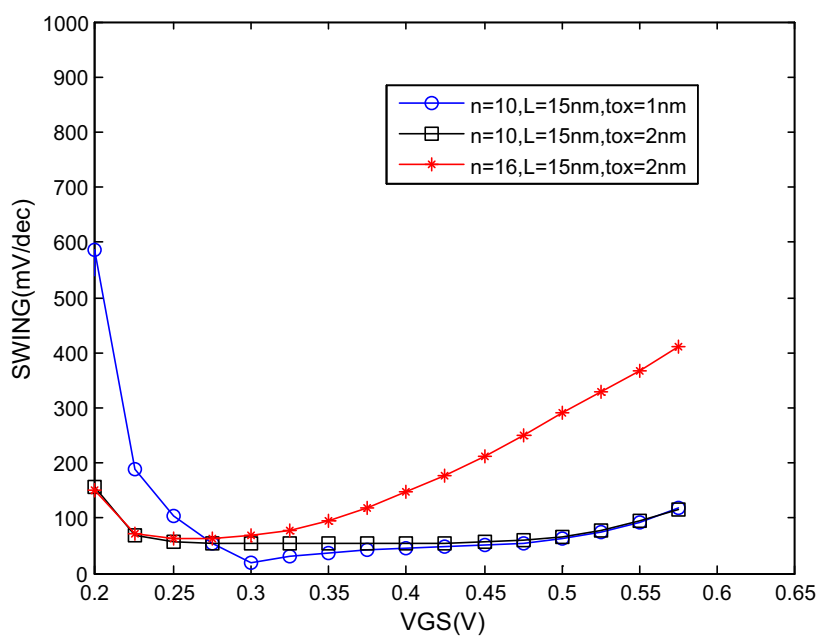

Fig. 13 Sub-threshold swing as a function of the gate-source voltage for $\operatorname{CNT}(10,0)$, tox $=1 \mathrm{~nm}$ and $(16,0)$, and tox $=2 \mathrm{~nm}$ at $V_{\mathrm{DS}}=0.4 \mathrm{~V}$

demonstrates that for various values of oxide thickness include in 2, 1.5 , and $1 \mathrm{~nm}$, SS achieves $62 \mathrm{mV} /$ decade which more than $60 \mathrm{mV} /$ decade conventional limits. To improve characteristics of tunneling carbon nanotube fieldeffect transistor, we select some channel parameters, and then investigate characteristics of the T-CNTFET regarding the selected parameters and compared to T-CNTFET with default parameters. As can be observed from Fig. 12, reduction channel diameter causes the reduction at off-current. For CNT with same diameter $(10,0)$, reduction oxide thickness leads to reduction at off-current that results in higher $I_{\mathrm{ON}} / I_{\mathrm{OFF}}$ ratio, nearly $>10^{7}$. In addition, sub-threshold swing of T-CNTFET with CNT $(16,0)$ and $(10,0)$ with various oxide thickness is compared in Fig. 13. It is evident 
from Fig. 13 that T-CNTFET with CNT $(10,0)$ with oxide thickness $=1 \mathrm{~nm}$ shows reduced sub-threshold swing (18 $\mathrm{mV} /$ decade) in comparison with T-CNTFET with CNT $(10,0)$ with oxide thickness $=2 \mathrm{~nm}$ and T-CNTFET with CNT $(16,0)$. Swing sub-threshold is decreased rather than [6]. This is attributed to control of the gate voltage of T-CNTFET $(10,0)$ with oxide thickness $=1 \mathrm{~nm}$ over the channel.

\section{Conclusion}

The NEGF formalism has been applied to simulate the electronic properties of the T-CNTFET structure with various channel parameters. The simulated characteristics were compared with those of the changing one of the channel parameter if other channel parameters are constant, the improved characteristics were discussed, finally. Simulations show that using CNT with larger bandgap leads to lower subthreshold swing and higher $I_{\mathrm{ON}} / I_{\mathrm{OFF}}$-current, while reducing on-current and tunneling current. In this paper, the highest value of the $I_{\mathrm{ON}} / I_{\mathrm{OFF}}$ ratio $\left(>10^{7}\right)$ and the least sub-threshold swing (18 mV/decade) observed by T-CNTFET with CNT $(10,0)$, oxide thickness $=2 \mathrm{~nm}$ and $\mathrm{CNT}(10,0)$, and oxide thickness $=1 \mathrm{~nm}$, respectively. Finally, simulation results demonstrate that changing the channel parameters alters characteristics of the devices, essentially.

Open Access This article is distributed under the terms of the Creative Commons Attribution 4.0 International License (http://crea tivecommons.org/licenses/by/4.0/), which permits unrestricted use, distribution, and reproduction in any medium, provided you give appropriate credit to the original author(s) and the source, provide a link to the Creative Commons license, and indicate if changes were made.

\section{References}

1. Paul, B.C., Shinobu, F., Masaki, O., Lee, T.: Modeling and analysis of circuit performance of ballistic CNTFET. Des. Autom. Conf. 41, 717-722 (2006)

2. Yong, Z.Z., Sheng, W., Lianmao, P.: High performance dopingfree carbon nanotube-based CMOS devices and integrated circuits. J. Chin. Sci. Bull. 57, 135-148 (2011)

3. Adessi, C., Avriller, R., Blasé, X., Boarnel, A., Cazin, H., Doiifus, P., et al.: Multiscaled simulation of carbon nanotube devices. C. R. Phys. E 10, 305-319 (2009)

4. Arefinia, Z.: Investigation of the performance and band-to-band tunneling effect of a new double-halo-doping carbon nanotube field-effect transistor. Phys. E 41, 1767-1771 (2009)

5. Sedigh-ziabari, S.A., Saghafi, K., Faez, R., Moravej-Farshi, M.K.: Nemerical investigation on temperature dependence of the cylindrical gate-all-around Si-nano wire FET. Mod. Phys. Lett. B 25, 2269-2278 (2011)

6. Koswatta, S.O., Nikonov, O., Lundstrom, M.S.: Computational study of carbon nanotube p-i-n Tunnel FET. Presented at Electron Devices Meeting. IEDM Technical Digest, pp. 518-521 (2005)

7. Naderi, A., Keshavarzi, P.: Novel carbon nanotube field effect transistor with graded Double halo channel. Sup. Micro 51, 668-679 (2012)

8. Arefinia, Z., Orouji, A.A.: Impact of single halo implantation on the carbon nanotube field-effect transistor. A quantum simulation study. Phys E 41, 196-201 (2008)

9. Graham, A.P., Deusberg, G.S., Hoenlein, W., Kruepl, F., Liebau, M., Martin, R.: How do you carbon nanotubes fits into the semiconductor Roadmap? Appl. Phys. Mater. Sci. Process 88, 1141-1155 (2005)

10. Lin, Sh., Kim, Y.B., Lombardi, F.: A new SRAM cell design using CNTFETs. In: Presented at International Conference SoC Design 1, pp. I168-I171 (2008)

11. Godoy, A., Lopez-Villanueva, J.A., Jimenez-Tejada, J.A., Palma, A., Gamiz, F.: A simple threshold swing model for short channel MOSFET. Solid State Electron. 45, 391-397 (2001) 